\title{
The attentional demands of negation in a memory-scanning task
}

\author{
JAMES H. HOWARD, JR. \\ Brown University, Providence, Rhode Island 02912
}

\begin{abstract}
Subjects were run in a Sternberg recognition memory task modified to occasionally require a transformation prior to response. The transformation was similar to logical negation since on plus or nontransformed trials subjects responded "yes" if the probe was from the current memory set and "no" otherwise, while on minus or transformed trials subjects responded "no" to memory set probes and "yes" to nonmemory set probes. Two models proposed to account for the effects of logical negation were compared: The encoding and comparison model predicts that logical negation requires an additional processing stage, while the capacity sharing model argues that negation adds a processing stage and also retards the rate of memory search. The results indicated that minus probes had $50 \%$ larger mean reaction time by set size slopes than the corresponding plus probes. This finding offers clear support for the capacity sharing model.
\end{abstract}

In recent years a number of studies have shown that logical negation serves to increase the processing time required in a variety of classification tasks (e.g., Chase \& Clark, 1972; Greene, 1970; Meyer, 1973; Trabasso, 1970; Wason, 1959). For example, Howard (1972) required subjects to classify logical symbols corresponding to the four information conditions discussed by Wason and Trabasso. Subjects responded "true" to $+\mathrm{T}$ and $-\mathrm{F}$ and responded "false" to $+\mathrm{F}$ and $-T$. The results of this experiment revealed that on the average, subjects required 140 msecs longer to classify the negative symbols $(-T,-F)$ than to classify the affirmative symbols $(+T,+F)$.

Most theories proposed to account for the effects of negation assume that classification involves four serial, independent processing stages: an encoding stage, a memory search stage, a comparison or decision stage, and a response stage. They assume that the total response time can be predicted from the algebraic sum of the durations of each individual processing stage. Since these models have emphasized the importance of the encoding and comparison stages, they have been referred to as encoding and comparison models.

Figure 1 displays the encoding and comparison model adapted from Trabasso (1970) to describe the processing of logical symbols (Howard, 1972). The model consists of four independent stages. In Stage 1 the subject encodes the stimulus into an internal representation. In Stage 2 the subject retrieves information about the response appropriate to the symbol ("true" or "false") and sets an internal "response index" equal to this value.

This report is based on part of the author's doctoral dissertation at Brown University. The author acknowledges the helpful comments of Peter Eimas, David Getty, Darlene Howard, David Meyer, Richard Millward, and Bryan Shepp on earlier versions of this manuscript.

This research was supported in part by Grant GB-34122 from the National Science Foundation to Richard B. Millward.

The author is now at the Department of Psychology. The Catholic University, Washington, D.C. 20017.
In Stage 3 the subject tests the encoded representation for the presence of negation and reverses the response index if a negative is present. In Stage 4 the response indicated by the response index is generated. According to this version of the model, the effect of negation is to increase the duration of the comparison stage by requiring a reversal of the response index.

Although most classification studies involving negation have supported some version of the encoding and comparison model, Meyer (1973) has pointed out that a number of the model's assumptions have not been explicitly tested. In particular, the model assumes that the memory retrieval process occurring in Stage 2 is independent of all other processing stages and is

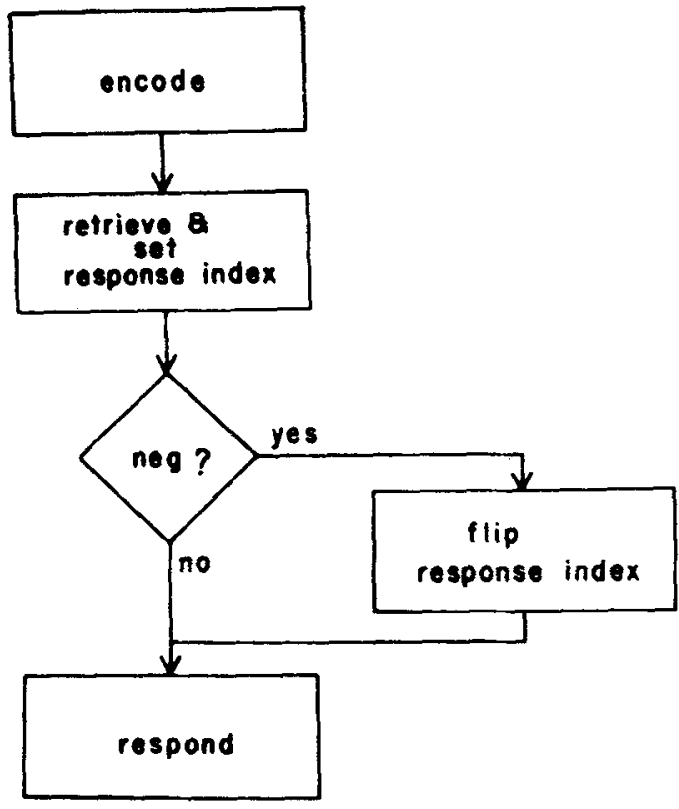

Figure 1. Flow chard of a simple information processing model for the classification of logic symbols. 
therefore not influenced by the presence of negation. Meyer argues that this assumption is not justified. $\mathrm{He}$ assumes that all processing, including the maintenance of information in active memory, is accomplished by a single, limited-capacity, central processing unit (e.g., Anderson \& Craik, 1974; Keele, 1973; Kerr, 1973; Moray, 1967; Posner \& Boies, 1971). As the demands on the central processor increase, the amount of time it can devote to each stage decreases. In this view, then, an increase in the total processing demands on the subject will necessarily influence any other ongoing processing. As the overall demands increase, the excess processing capacity available for other processing decreases.

Meyer has noted that most sentence classification models implicity assume that the internal representation of negative information, e.g., a sentence or logical proposition, is more complex than the internal representation of affirmative information (e.g., Clark, 1969). Consequently, he argues, negative information should require more processing capacity to maintain in active memory than affirmative information, thereby leaving less residual capacity for concurrent processing. Thus, the capacity sharing model predicts that the duration of the memory search stage should depend on the complexity of the internal representation produced in the encoding stage and, therefore, on the presence of negation. In other words, the capacity sharing model argues that the effect of negation is not strictly additive with the effects of other factors.

Some initial support for this position has been presented in a sentence verification experiment designed to test this assumption directly (Meyer, 1973). In this experiment, subjects were required to classify affirmative and negative sentences as "true" or "false" on the basis of general knowledge. Two factors that have been shown to affect memory retrieval time were varied in addition to negation (cf. Meyer, 1970). The results of the experiment indicated that these factors interacted with the effect of negation, a result supporting the capacity sharing model. Similar findings have been reported by Kreuger (1972) in a sentence-picture verification task. Overall, these data raise questions about the efficiacy of the encoding and comparison model.

Consider once again the encoding and comparison model outlined in Figure 1. Note that in the logical classification task the duration of the second, memory search stage is held relatively constant across experimental conditions. If, as the capacity sharing model assumes, negation influences the duration of the memory search stage, then any other experimental factor known to influence the duration of this stage should interact with the effect of negation (cf. Sternberg, 1966, 1969). In order to test this prediction, the logical classification task may be viewed as a special case of the Sternberg short-term recognition memory task. In the recognition memory task subjects are required to classify a series of letters (test probes) as old (i.e., members of a previously memorized "memory set") or new items (i.e., items not in the memory set). Sternberg has demonstrated that the duration of the memory search stage varies directly with the size of the memory set. According to Sternberg, the slope of the reaction time (RT) by set size function reflects the rate of memory search, and the intercept reflects the combined durations of all other processing stages.

In the present experiment subjects memorized sets of one, two, four, or six letters and were then given a series of test trials requiring either a "yes" or "no" response. On each trial, the subject was presented with a test letter and a code character, either a + or - . On trials where the code character was + (plus probes), the subject responded as in the Sternberg task: Yes if the letter was in the memory set, and no otherwise. On trials where the code character was - (minus probes), the subject reversed his response, responding no if the accompanying test letter was in the set and yes otherwise.

Since the encoding and comparison model assumes that negation has no effect on memory search (i.e., Stage 2), it predicts that plus and minus probes should differ by only an additive constant reflecting the additional time required to "flip" the response index in Stage 3. In other words, the encoding and comparison model predicts that the rate of memory scanning, and consequently the slopes of the RT by set size functions, should be the same for both plus and minus test probes. In contrast, the capacity sharing model argues that memory search is influenced by negation and, therefore, the effect of negation should interact with the effect of set size. Furthermore, since there would be less residual processing capacity available for memory search when negative information is being held in memory than when affirmative information is being held, the capacity sharing model predicts that minus probes should have steeper RT by set size slopes than plus probes. The predictions of the two models are displayed in Figure 2.

In addition to memory set size and sign (plus and minus), the exposure duration of the test probe was also varied in the present experiment. In one group, the indefinite presentation group, the test probe was displayed until the subject responded, while in the second group, the tachistoscopic presentation group, the test probe was presented for only $200 \mathrm{msec}$. The latter group was included as a control to insure that the subjects held both the sign and test letter in memory throughout the memory retrieval stage.

\section{METHOD}

\footnotetext{
Subjects

Subjects were four graduate and five undergraduate volunteers at Brown University who were paid for their participation.
} 


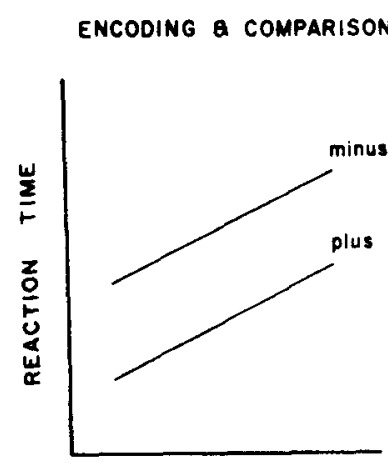

SET SIZE

\section{CAPACITY SHARING}

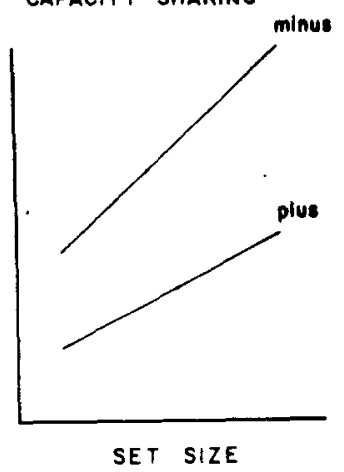

Figure 2. The idealized reaction time by set size functions for plus and minus probes predicted by the encoding and comparison and capacity sharing models.

\section{Apparatus}

The experiment was controlled by a program run on a PDP-8/L computer under the GASPS real-time programming system (Wickens, Howard, Rice, \& Millward, 1972). Each subject was seated in a sound-attenuated booth containing a standard Teletype keyboard with the "C" and "." keys relabeled "yes" and "no," and a 9-in. Shibaden television monitor driven by an Applied Digital Data Corporation MRD-200 alphanumeric character display. The television monitors were used to present the memory sets and test probes, and subjects responded to test probes by pressing a key on the keyboard. Test probes (black letters on a white background) subtended a visual angle of $2 / 3 \mathrm{deg}$ at a viewing distance of $18 \mathrm{in}$. and required a maximum presentation time of 1.2 msec. The computer recorded the subject's response and $\mathrm{RT}$ (the time from probe presentation to response accurate to within $1 \mathrm{msec}$ ) on magnetic tape for later analysis. The computer and data recording equipment were located in a room adjacent to the experimental room.

\section{Materials}

Individual memory sets were assembled by randomly sampling one, two, four, or six different characters from an alphabet of 25 letters (the letter $Q$ was omitted because of its similarity to $O$ on the television screens). A random sequence of 96 test probes was generated for each set. Of these, 48 probes were plus and 48 were minus. Half of the probes were sampled from the memory set (MS probes) and half were sampled from the remainder of the alphabet (NMS probes). Each of the four different types of probe (+MS, +NMS, -MS, -NMS) occurred 24 times, requiring an equal number of yes and no responses. Memory set probes (i.e., +MS and -MS) were selected, with the restriction that each serial position be tested an equal number of times. Consequently, individual memory set items were tested 24,12 , 6, or 4 times (for Set Sizes 1,2, 4 and 6, respectively) under both the plus and minus probe conditions.

\section{Design and Procedure}

Subjects were run either alone or independently in pairs for five 1 -h sessions. Sessions were divided into two blocks of 96 test trials each. In each block 24 test probes were presented for each of the four memory sets (i.e., a "fixed set" procedure was used, cf. Sternberg, 1969). Subjects responded to 768 probes per session for a total of 3,840 trials or 34,560 trials across the nine subjects. The first session was considered practice and the data were not included in the analysis.

At the beginning of each session, and whenever the memory set changed, the words NEW MEMORY SET were displayed to remind the subject that a new list had been selected. On request, the current memory set was displayed in a fixed serial order, with each letter remaining on the screen for $3 \mathrm{sec}$. Set items were never presented with either sign and the subjects could see the set as often as they wished between trials.

Five subjects pressed "yes" with the right hand and "no" with the left hand, while the response assignments were reversed for the remaining subjects. When the subject pressed space, the ready message was erased and after a random delay of 150 to $500 \mathrm{msec}$ a test probe was presented. For the indefinite presentation group the probe remained on the screen until the subject responded, and for the tachistoscopic presentation group the probe remained on the screen for a fixed period of $200 \mathrm{msec}$. Subjects were asked to respond "as quickly as possible without making errors." At the end of each block, the words THE END were displayed on the screen to signal the start of a 5-min break. After Session 5, each subject filled out a questionnaire concerning his performance in the experiment.

\section{RESULTS}

Mean correct RTs and error rates were computed for each factor for each subject in the experiment, and these data were used in all subsequent analyses. The overall error rates were $2.1 \%$ and $3.2 \%$ for the indefinite presentation and tachistoscopic presentation groups, respectively, with individual subjects ranging from $1 \%$ to $6.4 \%$. The error rates were considered sufficiently low to justify discarding the error RTs and these data were not considered further.

A preliminary four-way (set size, sign, MS/NMS, and presentation) repeated measurements analysis of variance on the mean correct RTs for each condition revealed no significant main effect of mode of presentation, $F(3,24)<1$, and no significant interactions between mode of presentation and any of the other experimental factors. These results suggest that subjects in both groups were processing the test probes in a similar manner, and all subsequent analyses were performed on the combined data from the two groups.

Mean RT by set size plots for each of the four different probe conditions (i.e., +MS, +NMS, $-\mathrm{MS}$, -NMS) are displayed in Figure 3, and the mean RTs for each point are presented in Table 1. A four-way (set size, sign, MS/NMS, and session) repeated measurements analysis of variance with trend evaluation on the set size factor (Myers, 1967, Chap. 14) revealed several significant effects in these data. Three main effects (terms containing the session factor will be discussed later), set size $[F(3,24)=44.66, p<.001]$, sign $[F(1,8)=31.71, \quad p<.001]$, and $\mathrm{MS} / \mathrm{NMS}$ $[F(1,8)=22.64, p<.005]$, and two interactions, Set Size by Sign $[F(3,24)=11.02, p<.001]$ and MS/NMS by Sign $[F(1,8)=39.88, p<.001]$, reached statistical significance. Each of these effects is examined in more detail below.

Further analysis of the set size effect revealed that linear trend accounted for $99.7 \%$ of the variance in the effect, $F(1,8)=51.66, p<.001$. This result, together 


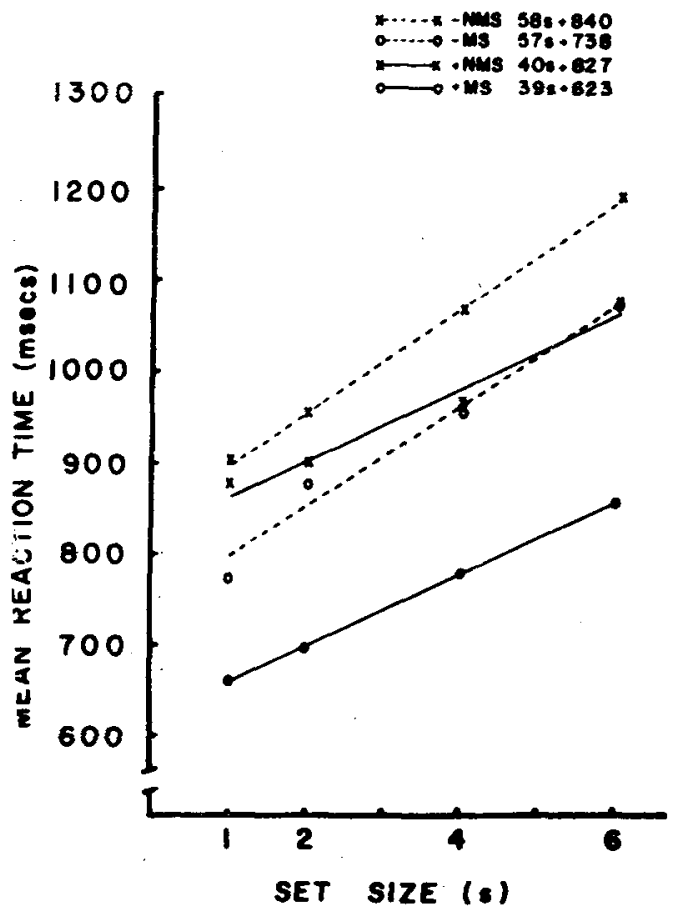

Figure 3. Observed and best-fitting mean reaction time by set size functions for the four probe conditions.

with the absence of any higher order trend, $F(2,16)<1$, suggests that linear functions with nonzero slopes provide the best description of the set size effect. Linear equations were fit to the data by the method of least squares, and these functions are also displayed in Figure 3. The MS/NMS factor had no effect on the slope of these functions, $F(3,24)=1.01$; however, the sign factor had a large effect, with minus probes producing 50\% larger slopes than the plus probes, $F(1,8)=13.89, p<.01$. This finding is clearly consistent with the predictions of the capacity sharing model and is inconsistent with the additivity assumption of the encoding and comparison model.

A second effect of some interest is that minus probes required an average of $122 \mathrm{msec}$ longer to process than plus probes. This result suggests that the minus transformation increases processing demands in the present task in a way similar to negation in the sentence verification and symbol classification tasks. In addition, the slope difference reported above indicates that the effect of minus was not constant, but increased with increasing set size. Minus memory probes required an additional $67,116,140$ and $167 \mathrm{msec}$ of processing time for set sizes of $1,2,4$, and 6 , respectively. Once again, this result is not consistent with the encoding and comparison model's prediction that the effect of the minus transformation should be constant across conditions.

A third finding was that NMS probes required an average of $158 \mathrm{msec}$ longer than MS probes. Although this effect was constant across the different set sizes, the MS/NMS factor did interact significantly with sign. This interaction was examined further by testing the pairwise differences in mean RT for each of the four probe conditions with Duncan's new multiple-range test (Edwards, 1968). This analysis revealed the following differences in mean RT: +MS $(751 \mathrm{msec})<-$ MS $(921)=+$ NMS $(957)<-$ NMS $(1031)$ (all tests were run at the .01 level, yielding an overall protection level of .03). The rank ordering of these data is consistent with the overall processing scheme suggested by the encoding and comparison model and the assumption that subjects initiate processing with the response index set to "yes." Under these conditions the subject would have to invert the response index for both minus and NMS probes, resulting in one inversion for $-\mathrm{MS}$ and + NMS probes and two inversions for the -NMS probes. Accordingly, the encoding and comparison model predicts that the total response time for-NMS probes should reflect the base response time (as indicated by the +MS probes = $751 \mathrm{msec}$ ) plus the additional time for minus (-MS less $+\mathrm{MS}=170 \mathrm{msec}$ ) and NMS probes (+NMS less +MS = $206 \mathrm{msec}$ ). A closer examination of this interaction in the present experiment revealed that the RT predicted by the additive encoding and comparison model for the -NMS condition $(1,127 \mathrm{msec})$ was too large by about $100 \mathrm{msec}, \mathrm{t}(8)=6.23, \quad \mathrm{p}<.001$. This discrepancy indicates that the sign and MS/NMS effects are clearly not additive, a finding inconsistent with the predictions of the encoding and comparison model.

The session factor was included in the analysis to determine if the results reported above were stable across the four experimental sessions. This analysis revealed a significant main effect of session $[F(3,24)=11.68, p<.001]$ and significant Set Size by Session $[F(9,72)=3.92, p<.001]$ and Set Size by Sign by $M S / N M S$ by Session $[F(9,72)=2.39, p<.05]$ interactions. No other interactions with session were

Table 1

Mean Reaction Time and Percent Error for Each Experimental Condition

\begin{tabular}{|c|c|c|c|c|c|c|c|c|}
\hline \multirow{2}{*}{$\begin{array}{l}\text { Probe } \\
\text { Type }\end{array}$} & \multicolumn{2}{|c|}{ Set Size 1} & \multicolumn{2}{|c|}{ Set Size 2} & \multicolumn{2}{|c|}{ Set Size 4} & \multicolumn{2}{|c|}{ Set Size 6} \\
\hline & RT & Error & $\mathrm{RT}$ & Error & RT & Error & $\mathrm{RT}$ & Error \\
\hline$+\mathrm{MS}$ & 665 & 1.0 & 698 & 1.5 & 779 & 2.8 & 860 & 2.9 \\
\hline +NMS & 878 & 2.6 & 902 & 1.4 & 969 & 1.7 & 1078 & 2.0 \\
\hline -MS & 776 & 2.8 & 878 & 3.2 & 955 & 2.6 & 1077 & 4.6 \\
\hline -NMS & 903 & 2.6 & 954 & 3.5 & 1072 & 3.9 & 1194 & 4.3 \\
\hline
\end{tabular}


significant. The four-way interaction had no obvious interpretation in this experiment and, therefore, it was not considered further. The main effect of session was produced by a decrease in the overall mean RT across sessions. A tendency for this improvement in mean RT (52, 35, and $15 \mathrm{msec}$ for Sessions 3, 4, and 5, respectively) to decrease as the subjects became more experienced suggests a general practice effect. Trend analyses on the set size factor revealed that most of the Set Size by Session interaction $(88.9 \%)$ could be accounted for by a significant linearity of set size by session component, $F(3,24)=6.01, \mathrm{p}<.005$; the higher order components were not significant. This finding indicates that the slopes of the RT by set size functions were decreasing over experimental sessions. Most importantly, however, there was no indication that the difference between plus and minus slopes was changing with practice, and the overall improvement in RT leveled off by Session 4 . In general, then, it appears that the findings reported above are reliable across experimental sessions.

The contingent error rates for each experimental condition across all subjects are presented in Table 1 . Extensive analyses were not performed on these data because overall error rates were very low and generally unreliable. However, a Spearman rank-order correlation between mean percent errors and mean RT indicated that error rate tended to correlate positively with mean $\mathrm{RT}, \mathrm{r}=.46, \mathrm{p}<.05$. The overail error rates were $2.3 \%$, $2.4 \%, 2.7 \%$, and $3.4 \%$ for set sizes of $1,2,4$, and 6 items, respectively, and in all cases minus probes had higher mean error rates than plus probes. Further analyses indicated that the group data reported above provide an accurate description of the individual subject data as well.

\section{DISCUSSION}

Overall, the results reported above support the capacity sharing model. The effect of the minus transformation was observed to increase with increasing set size. In terms of Sternberg's analysis, this result indicates that the rate of memory scanning is slower for minus probes than for plus probes. This finding cannot be predicted by the additive model. However, at least two alternative explanations of this result do not require the capacity sharing assumption.

The first possibility is that subjects process the probes in a strictly sequential fashion, but that on some proportion of the minus probe trials they search the memory set twice to "double check" the initial scan. It is conceivable that this rechecking is prompted by an incompatibility between the transformed and "natural" response tendencies. This explanation would predict the observed slope difference between minus and plus probes. However, it also predicts that the RT distributions for the minus probes should be bimodal. The RT distributions for individual subjects were examined in detail, and although in general the minus distributions tended to be flatter than the plus distributions, no consistent evidence was obtained in support of this hypothesis.

A second possibility is that subjects encode the items in the memory set as visual images of plus probes; that is, as the set letters each .preceded by a + sign. ${ }^{1}$ During the comparison stage, an image of the test probe could be compared with each of the members of the memory set. Under these conditions, a minus test probe would differ from all of the set items in terms of its sign. If it is assumed that finding mismatching signs requires more time than finding matching signs, then the memory search stage should be slower for minus probes than for plus probes, and consequently the slopes should be steeper for minus than for plus probes. Although this explanation cannot be completely ruled out, it may be criticized on at least two grounds. First, there is no a priori reason to assume that the memory set items should be encoded as plus probes since they were not presented with either sign. Second, each memory set item for subjects using this strategy would contain more information than each item in a standard Sternberg task. Given this difference, memory scanning for plus probes in the present experiment would be expected to be slower than in the standard task (e.g., Cavanagh, 1972). This prediction is inconsistent with the findings of the present experiment since scanning rates in the present experiment were identical to Sternberg's.

Overall, then, it appears that the capacity sharing model offers the most reasonable explanation of the present results. Several aspects of the data make this conclusion particularly convincing. (1) The data obtained for the plus probes, the condition which most closely approximates the standard recognition memory task, are generally consistent with the results reported by Sternberg (1966) and others. Although the intercepts in the present study were somewhat larger than are typically obtained, probably reflecting a general task difference, the overall slopes reported above are representative of earlier results with alphabetic characters. Swanson, Johnsen, and Briggs (1972) have reported similarly large intercepts in a memory scanning task where subjects were required to perform a transformation prior to response. Further evidence for a difference in task complexity is reflected in the tendency for the RT by set size slopes to decrease across experimental sessions. Initially, i.e., Sessions 2 and 3, the slopes were larger than usual; however, Sessions 4 and 5 produced slopes consistent with previous experimental work. In addition, the slopes for corresponding MS and NMS probes support the contention that the addition of the sign factor to the memory scanning task did not substantially influence the overall processing strategies 
used by the subjects, and lend further support to the conclusion that memory search occurs more slowly for minus than plus probes. (2) The 50\% decrease in scanning rate observed for minus probes in the present study is consistent with Meyer's (1973) observation that long-term memory retrieval required about $50 \%-75 \%$ longer for negative sentences than for affirmative sentences. This similarity suggests that the capacity sharing model may apply equally well to long-term and short-term memory tasks. (3) The slope difference observed between plus and minus probes was shown to be robust over experimental sessions despite an overall tendency for the slopes to decrease through Session 4 . This practice effect is consistent with, although somewhat larger than, results reported by a number of studies in the literature (e.g., Briggs \& Blaha, 1969; Ross, 1970 ) and probably reflects the development of more efficient search strategies.

\section{REFERENCES}

Anderson, C. M. B., \& Craik, F. I. M. The effect of a concurrent task on recall from primaly memory. Journal of Vexbal Learning and Verbal Behavior, 1974, 13, 107-113.

Briggs, G. E., \& Blaha, J. Memory retrieval and central comparison times in information processing. Journal of Experimental Psychology, 1969, 79, 395-402.

Cavanagh, J. P. Relation between the immediate memory span and the memory search rate. Psychological Review, 1972, 79, 525-530.

Chase, W. G., \& Clark, H. H. Mental operations in the comparison of sentences and pictures. In L. Gregg (Ed.), Cognition in learning and memory. New York: Wiley, 1972.

Clark, H. H. Linguistic processes in deductive reasoning. Psychological Review, 1969, 76, 387-404.

Edwards, A. L. Experimental design in psychological research. New York: Holt, Rinehart and Winston, 1968.

Greene, J. M. The semantic function of negatives and passives. British Journal of Psychology, 1970, 61, 17-22.

Howard, J. H. A reaction time analysis of simple symbolic logic. Unpublished Master's thesis, Brown University, 1972.

Howard, J. H. Information processing demands in a short-term memory task. Technical Report No.12, 1973. Studies in human learning. Psychology Department, Brown University, Providence, R.I.
Keele, S. W. Attention and human performance. Pacific Palisades: Goodyear, 1973.

Kerr, B. Processing demands during mental operations. Memory \& Cognition, 1973, 1, 401-412.

Krueger, L. E. Sentence-picture comparison: A test of additivity of processing time for feature matching and negation coding. Journal of Experimental Psychology, 1972, 95, 275-284.

Meyer, D. E. On the representation and retrieval of stored semantic information. Cognitive Psychology, 1970, 1 , 242-300.

Meyer, D. E. Verifying affirmative and negative propositions: Effects of negation on memory retrieval. In $\mathrm{S}$. Kornblum (Ed.), Attention and performance IV. New York: Academic Press, 1973.

Moray, $N$. Where is capacity limited? A survey and a model. Acta Psychologica, 1967, 27, 84-92.

Myers, J. L. Fundamentals of experimental design. Boston: Allyn and Bacon, 1967.

Posner, M. I., \& Boies, S. J. Components of attention Psychological Review, 1971, 78, 391-408.

Ross, J. Extended practice with a single-character classification task. Perception \& Psychophysics, 1970, 8, 276-278.

Sternberg, S. High-speed scanning in human memory. Science, $1966,153,652 \cdot 654$.

Sternberg. S. The discovery of processing stages: Extensions of Donder's method. Acta Psy chologica, 1969, 30, 276-278.

Swanson, J. M., Johnsen, A. M., \& Briggs, G. E. Recording in a memory search task. Journal of Experimental Psychology, $1972,93,1-9$.

Trabasso, T. Reasoning and the processing of negative information. Invited address, Division 3, American Psychological Association, 1970.

Trabasso, $\mathrm{T}$. Mental operations in language comprehension. In $\mathrm{J}$. B. Carroll and R. Freedle (Eds.), Language comprehension and the acquistion of knowledge. Washington, D.C: Winston, 1972.

Wason, $P, C$. The processing of positive and negative information. Quarterly Journal of Experimental Psychology, $1969,11,92-107$.

Wickens, T. D., Howard, J. H., Rice, G. A., \& Millward, R. B. GASPS: A general asynchronous processing system for the PDP-8. Behavior Research Methods \& Instrumentation, 1972, 4, 108-109.

\section{NOTE}

1. The author thanks David E. Meyer for suggesting this alternative.

(Received for publication July 26, 1974; revision accepted September $7,1974$. 